

\title{
Simulation and Compensation of Multibunch Energy Variation in NLC*
}

\author{
K. A. Thompson and R. D. Ruth \\ Stanford Linear Acceberutor Center, Stanford Unumersaty. Stanford, CA 94309 USA
}

Abstract

The SLAC NLC design for a next-generation linear collider utilizes multibunching (arceleration of a train of bunches on each RF fill) to increase the luminosity and energy efficiency. It is necessary to control the energy spread of the beam, in order to minimize chromatic emittance dilution and be within the energy acceptance of the final focus. It is anticipated that the NLC may run with bunch trains having length equal to a substantial fraction of the filling time. Multibunch energy simulation methods and compensation schemes appropriate to this regime are presented.

\section{INTRODUCTION}

Utilizing multibunching in a next-generation linear collider (NLC) requires that the energies of the bunches be tightly controlled. To be within the acceptance of the final focus system and to control chromatic emittance dilution in the linac, $\delta E / E$ needs to be less than about $0.15 \%$.

By adjusting the timing of injection of the bunch train with respect to the RF pulse, and choosing the bunch spacing appropriately, one may cancel most of the energy variation between bunches in the train. The basic idea is to have the RF structure fill with sufficient extra energy between bunch passages to make up for the energy lost in accelerating the preceding bunches in the train.

However, with the simplest form of this "matchedfilling" scheme [1], there is a "sag" in energy at the middle of the bunch train, and the longer the bunch train the greater the sag. In this paper we shall focus on compensation that permits running longer bunch trains $(\sim$ a filling time) while maintaining an acceptable energy spread. Long trains are under consideration as a way of obtaining the maximur. possible luminosity and energy efficiency.

We begin by discussing the factors that affect the energy spread of the beam. A detailed simulation program has been written, the elements of which are outlined here. In this simulation, one may take account of input RF pulse shaping and timing, the dispersion of the RF pulse as it transits the structure, the longitudinal distribution of charge within the bunches, the long range wake (LRW) including both the fundamental (accelerating) mode and higher order modes (HOM's), the short range wake (SRW), and phasing of the bunches with respect to the crests of the RF. We shall focus only on the inter-bunch energy spread in the present paper.

* Work supported by Departuent of Encrgy contract DE ACO3 TCSFO(1515

\section{BASIS OF SIMULATION}

We begin by considering a single accelerating section, fed at its upstream end by an mput $R F$ waveform that travels to the other end and is absorbed in a load. At sone specified tine with respect to the entry of the RF pulse, a train of relativistic $(v=c)$ bunches enters the structure, and the electrons in each bunch are accelerated by the fields (sum of RF pulse and wake fields) they encounter in the structure. The total charge in each bunch is divided into a finite number of longitudinal slices, small enougr that the longitudinal position and the energy gain of the electrons in a given slice may be taken to be equal.

The total voltage gained in the section by slice $r$ of bunch $n$ may be broken down inte

$$
\Delta V_{n, r}=\Delta V_{n, r}^{r f}+\Delta V_{n, r}^{l r w}+\Delta V_{n, r}^{s r w}
$$

We denote the time of entry of this slice into the section by $t_{n, r}$, and longitudinal position in the section by $s$, where $s$ runs from 0 to the structure length $L$, and consider each of these three contributions to the total section voltage gain.

The voltage $\Delta V_{n, r}^{r f}$ due to the RF pulse is

$$
\Delta V_{n, r}^{r f}=\int_{0}^{L} E^{r j}\left(s, t_{n, r}+s / c\right) d s
$$

where $E^{r f}(s, t)$ is the field of the RF waveform at location $s$ and time $t$, obtained by propagating the input RF pulse $E^{r f}(0, t)$ down the structure. Iet us specify the input RF pulse as

$$
E^{r f}(0, t)=\hat{E}_{0}(t) \cos \left(\omega_{r f} t+\varphi_{0}\right)
$$

The accelerating frequency $\omega_{r f}$ is assumed to have phase velocity in the structure equal to $c$ and thus is synchronous with the charges to be accelerated.

If we neglect dispersion, i.e., assume the group velocity $v_{g}$ is the same for all frequency components in the pulse (though it may in general still depend on $s$ ), we have

$$
\begin{aligned}
E^{r f}(s, t) & =\hat{E}_{0}\left(t-\int_{0}^{s} \frac{d s^{\prime}}{v_{g}\left(s^{\prime}\right)}\right) \\
& \cdot \exp \left(-\int_{0}^{s} \alpha\left(s^{\prime}\right) d s^{\prime}\right) \cos \left[\omega_{r f}(t-s / c)+\varphi_{0}\right] .
\end{aligned}
$$

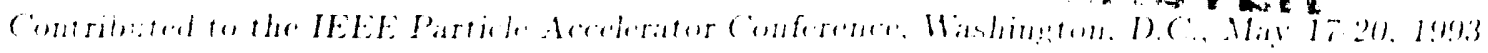


In reality d spersion mates the shape of the kil pular change as it propagates through the structuse partich larly for a sharply varying esvelope $\hat{E}_{0}(t)$. Thus, une should Fourier analyze the ingu pulse,

$$
E(\omega)=\int_{-\infty}^{\infty} E^{v f}(0, t), \cdots i \omega^{\prime} t d t
$$

and propagate each of its frequency components according to the dispersion relation $\Gamma(\omega, s)=\alpha(\omega, s)+i / \gamma(\omega, s)$ for the structure:

$$
E^{r f}(s, t)=\frac{1}{2 \pi} \int_{-\infty}^{\infty} E(\omega) \exp \left[i \omega t-\int_{0}^{s} \Gamma\left(\omega, s^{\prime}\right) d s^{\prime}\right] d \omega
$$

Here we assume that the structure can be modelled locally as a band-pass filter, though its properties may change gradually with $s$.

For a narrow-band structure with small attenuation [2], $\alpha$ may be assumed independent of $\omega$, and a good approximation to $\beta(\omega, s)$ is

$$
\beta(\omega, s)=\frac{1}{d} \cos ^{-1}\left[-\frac{\omega-\omega_{0}(s)}{\omega_{H_{u}}(s)}\right],
$$

where $d$ is the structure period (cell length), $\omega_{0}(s)$ is the mid-band frequency, and who (s) the half-width of the passband. In the usual situation, the accelerating mode lies in the range of $\beta$ between 0 and $\pi / d$; it is common to choose $\beta\left(\omega_{r f}, s\right) d=\frac{2 \pi}{3}$.

The long range wake voltage $\Delta l_{n, r}^{r \text { ru }}$ folt by the slice is

$$
\Delta V_{n, r}^{l r u}=\sum_{j=1}^{n-1} \int_{0}^{L} E^{l r u}\left(s, t_{n, r}+s / c, j\right) d s
$$

where $E^{l w}(s, t, j)$ is the field at location $s$ and time $t$, due to the wake left by bunch $j$.

There are two simpler special cases of particular interest. One is the constant-impedance (CZ) structure, made of identical cells so that its properties such as $v_{g}$, a, ete., are independent of $s$. For the CZ structure, we have

$$
\begin{aligned}
E^{r \rho}(s, t) & =\frac{1}{\pi} \int_{\omega / 0}^{\omega_{\omega p}}[\operatorname{Re} E(\omega) \cos [\omega t-\beta(\omega) s] \\
& -\ln E(\omega) \sin [\omega t-\beta(\omega) s]] \sigma^{-\alpha(\omega) s} d \omega
\end{aligned}
$$

The ofleer special case is the comstant-gradient ( $(\mathrm{C})$ structure. which when unleaded has constant electric field alemet the strueture when led with a combtam ampli.

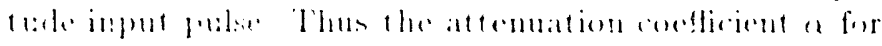



$$
r_{y}(s)=\frac{u g}{u}\left[L^{\prime} \cdot s\right]
$$

where $L^{\prime}=\frac{l}{1}$, We $^{2}$ assumer (shunt impedance per unit length) and $Q$ are approximately independent of $s$ For the CG structure,

$$
\begin{aligned}
E^{r f}(s, t) & =\frac{1}{\pi} \int_{\omega_{t}^{\prime}(s)}^{\omega_{u r(s)}^{u(s)}}[\operatorname{Re} E(\omega) \cos [\omega t-B(\omega, s)] \\
& -\ln E(\omega) \sin [\omega t-B(\omega, s)]] e^{-\alpha(\omega) s} d \omega
\end{aligned}
$$

where $B(\omega, s)$ may be derived analytically [3]. The limits $\omega_{l o}$ and $\omega_{u p}$ are the lower and npper passhand boundaries of the fundamental mode. The $\mathrm{CG}$ structure is a fairly good representation of the detuned structures being contemplated for the SLAC NIC design.

Denoting the time when bunch $j$ enters the structure by $t_{j}^{e n t}$, and neglecting dispersion, we have for the LRW voltage in the $\mathrm{C} Z$ casse:

$$
\begin{aligned}
E^{l r w}(s, t, j) & =\sum_{m}-2 r_{m} q_{j} F_{m, j} \exp \left[-\left(t-t_{j}^{c n t}-\frac{s}{c}\right) \frac{\omega_{m}}{2 Q_{m}}\right] \\
& \cdot \cos \left[\omega_{m}\left(t-t_{j}^{n t}-\frac{s}{c}\right)\right] \\
& \cdot H\left(t-t_{j}^{c n t}-\frac{s}{c}\right) H\left(t_{j}^{r n t}+\frac{s}{v_{g, m}}-t\right) .
\end{aligned}
$$

Here the sum $m$ rums over the modes in the wake (fundamental and HOM's); $\kappa_{m}$ is the loss factor, $Q_{m}$ the quality factor, and $v_{y, m}$ the group velocity of mode $m$. $F_{m, j}$ is a form factor that depends on the charge distribution of bunch $j$; for a Gaussian distribution, $F_{m, j}=$ $\exp \left(-\omega_{m}^{2} \sigma_{t, j}^{2} / 2\right)$. In future linear collider designs, the bunch length is very short, and we may take $F_{m, j}=1$. $H(t)$ is the unit step function.

The contribution of the fundamental mode to the LRW in the C:G rase is

$$
\begin{aligned}
E_{1}^{l r u^{\prime}}(s, t, j)= & -2 \kappa_{1} q_{j} F_{1, j} \cos \left[\omega_{1}\left(t-t_{j}^{e n t}-\frac{s}{c}\right)\right] \\
& \cdot H\left(t-t_{j}^{e n t}-\frac{s}{c}\right) H\left(t_{j}^{e n t}+t_{s}-t\right)
\end{aligned}
$$

where

$$
t_{s}=-\frac{Q_{r f}}{\omega_{r f}} \ln \left(1-\frac{s}{L^{\prime}}\right),
$$

and $\kappa_{1}$ is assumed to be independent of s. The HOM's in non-CZ structures may be treated by equivalent circuit models [4]. Lut this is hegend the serege of the present paper. 
Table 1: Parameters

RF frequency, $f_{r f}=11.424 \mathrm{GHz}$

Section length $=1.8 \mathrm{~m}$

Attenuation $\tau=0.505$

Fundamental mode $Q=7107$

Fund. mode loss factor, $\kappa_{1}=203.75 \mathrm{~V} / \mathrm{pC}$

Filling time, $T_{f}=100 \mathrm{~ns}$

Bunch spacing $=16 \lambda_{r f} \approx 42 \mathrm{~cm}$

Bunch charge $=1 \times 10^{10}$

\section{COMPENSATION SCHEMES AND EXAMPLES}

We model the linac as made up of CG sections (as defined above), with $2 \pi / 3$ phase advance per cell. bunch charge $=1 \times 10^{10}$. Parameters are as shown in Table 1 .

The most promising strategy useful for bunch trains of length a filling time or longer, is to pre-fill the structure in such a way that the energy gain of each bunch during the transient period approximates the energy gain of each bunch in the steady state [5]. In the simplest form of this scheme, the amplitude of the input $R F$ field $[-2$ is linearly ramped during the first filling time, then the bunch train is injected at the beginning of the second filling time. The input $R F$ pulse used in our example is [I] shown in Fig. 1. However, since dispersion creates large ${ }^{0}$ amplitude variations on the front of the $R F$ pulse, it is desirable to wait an additional $10 \mathrm{nsec}$ before injecting the bunch train, to allow the worst of these dispersion "wiggles" to propagate out of the structure. The resulting fractional energy deviation, for a 90-bunch train is shown in Fig. 2. Were it not for the effects of dispersion, the steady state would be reached at about bunch 70, as can be seen in Fig. 2(a), where dispersion is not included in the calculation. Fig. 2(b) shows the result with dispersion included, but with its effects diminished by allowing the front $10 \mathrm{nsec}$ of the pulse to propagate out of the structure before injecting the bunch train.



Figure 1. Envelope of input RF pulse, used in long-pulse pre-filling compensation scheme.

Another possible scheme for long bunch trains involves staggering the timing of the $\mathrm{RF}$ pulses in different sections [6]. Space prevents a detailed discussion of this scheme here; the overall energy compensation (net effect over a sum of sections) obtained with this scheme is similar to that of the preceding scheme. It does not, however, keep the energy as well compensated locally.

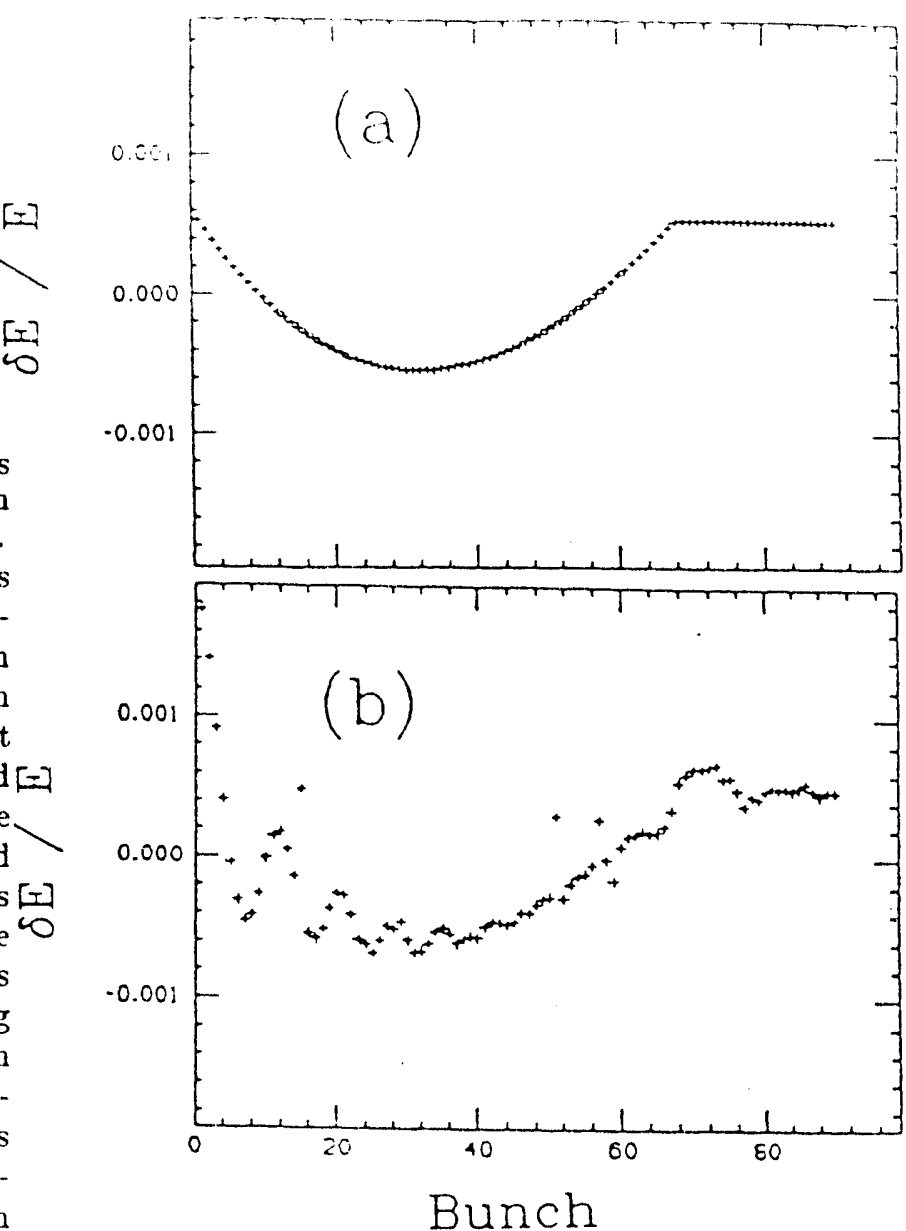

Figure 2. Fractional energy deviations of bunches in longpulse pre-flling compensation scheme, (a) ignoring dispersion, (b) including dispersion.

Finally, we mention a related strategy [7], in which one approximately equalizes the bunch energies via the matched-filling method, and then modulates the RF input during the time when the train is passing through the structure, to compensate the "sag" one would otherwise get in the middle of the bunch train. Such a scheme may be the best for irains which are of order a half of the filling time in length.

We thank D. Farkas, R. Miller, and R. Palmer for suggestions regarding compensatic: schemes, and the members of the NLC structures group for other helpful discussions.

[1] R.D. Ruth, in Proceedings of ICFA/INFN Workshop on Physics of Linear Colliders, Capri, Italy (1988), and SLAC-PUB-4541.

[2] J.Leiss, in Linear Accelerators, edt. by P.Lapostolle and A.Septier, (North-Holland, 1970).

[3] K.A. Thompson and R.D. Ruth, to be published.

[4] K.Bane, private communication.

[5] R.Palmer and R.Miller, private communication.

[6] R.Miller, private communication.

17) Z.D. Farkas, 16th International Linac Conference, Ottawa, Ontario, (23-28 August 1992); SLAC-PUB-5879. 


\section{DISCLAIMER}

This repor: was prepared as an account of work sponsored by an agency of the United States Governmellt. Neither the United States Government nor any agency thereof, nor any of their employees, makes any warranty, express or implied, or assumes any legal liability or responsibility for the accuracy, completeness, or usefulness of any information, apparatus, product, or process disclosed, or represents that its use would not infringe privately owned rights. Reference hereir to any specific commercial product, process, or service by trade name, trademark, manufacturer, or otherwise does not necessarily constitute or imply its endorsement, recommendation, or favoring by the United States Government or any agency thereof. The views and opinions of authors expressed herein do not necessarily state or reflect those of the United States Government or any agency thereof. 

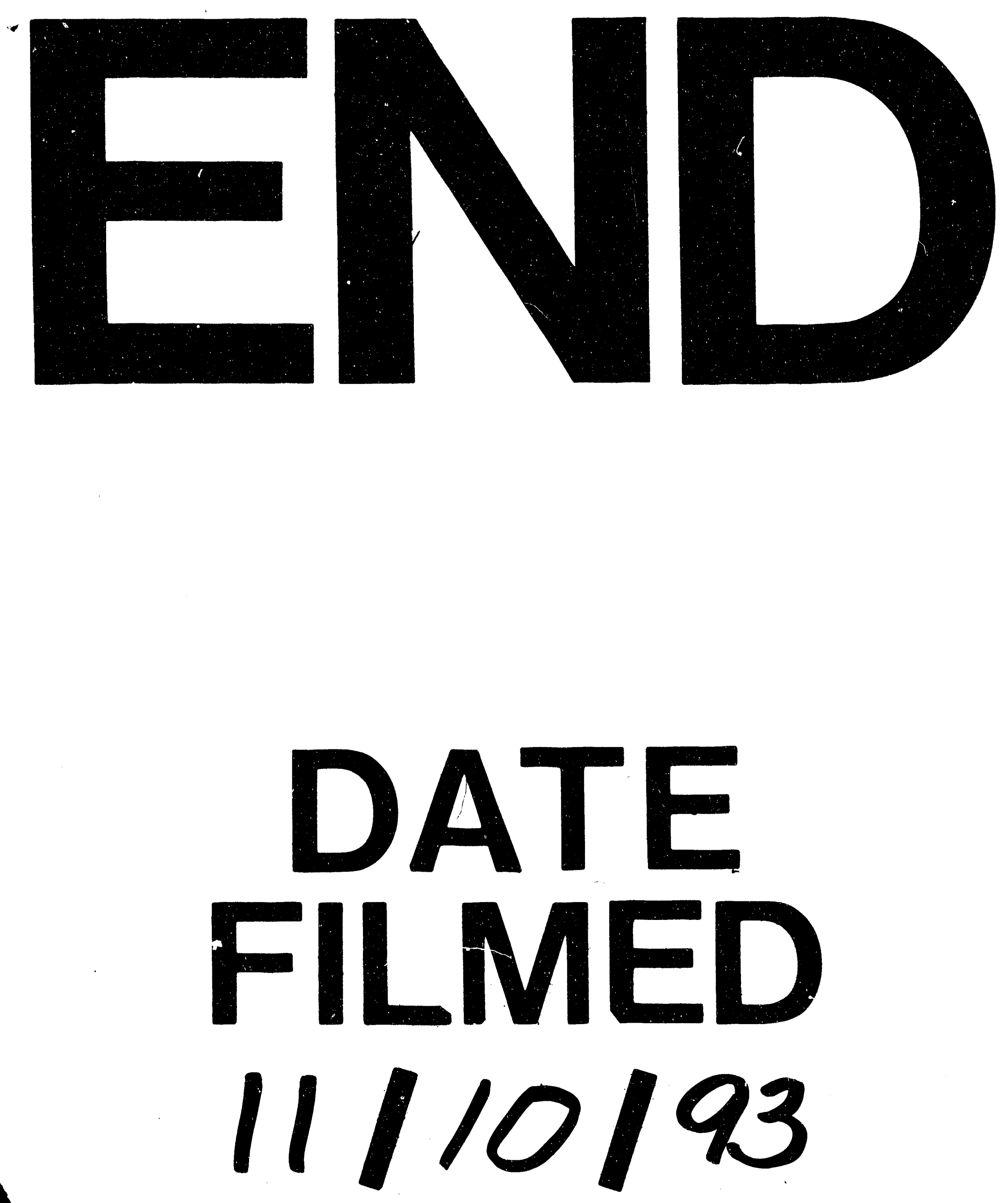
